UDC 517.9

R. Hakl (Math. Inst. Acad. Sci. Czech Republic, Brno)

PERIODIC BOUNDARY-VALUE PROBLEM

FOR THIRD ORDER LINEAR

FUNCTIONAL DIFFERENTIAL EQUATIONS*

ПЕРІОДИЧНА ГРАНИЧНА ЗАДАЧА

ДЛЯ ЛІНІЙНИХ ФУНКЦІОНАЛЬНО-ДИФЕРЕНЦІАЛЬНИХ

РІВНЯНЬ ТРЕТЬОГО ПОРЯДКУ

For the linear functional differential equation of the third order

$$
u^{\prime \prime \prime}(t)=\ell(u)(t)+q(t)
$$

theorems on the existence and uniqueness of a solution satisfying the conditions

$$
u^{(i)}(0)=u^{(i)}(\omega), \quad i=0,1,2,
$$

are established. Here, $\ell$ is a linear continuous operator transforming the space $C([0, \omega] ; R)$ into the space $L([0, \omega] ; R)$, and $q \in L([0, \omega] ; R)$. The question on the nonnegativity of a solution of the considered boundary-value problem is also studied.

Для лінійного функціонально-диференціального рівняння третього порядку

$$
u^{\prime \prime \prime}(t)=\ell(u)(t)+q(t)
$$

встановлено теореми про існування та єдиність розв'язку, що задовольняє умови

$$
u^{(i)}(0)=u^{(i)}(\omega), \quad i=0,1,2 .
$$

Тут $\ell €$ лінійним неперервним оператором, що трансформує простір $C([0, \omega] ; R)$ у простір $L([0, \omega] ; R)$, a $q \in L([0, \omega] ; R)$. Також розглянуто питання про невід'ємність розв'язку розглядуваної граничної задачі.

Introduction. Consider the linear functional differential equation

$$
u^{\prime \prime \prime}(t)=\ell(u)(t)+q(t)
$$

where $\ell: C([0, \omega] ; R) \rightarrow L([0, \omega] ; R)$ is a linear continuous operator and $q \in L([0, \omega] ; R)$.

By a solution of the equation (0.1) we understand a function $u:[0, \omega] \rightarrow R$, which is absolutely continuous together with its first and second derivatives and satisfies the equation $(0.1)$ almost everywhere in $[0, \omega]$.

The following notation will be made use of:

$$
\begin{aligned}
R & =]-\infty,+\infty[, & R_{+} & =[0,+\infty[, \\
{[x]_{+} } & =\frac{1}{2}(|x|+x), & {[x]_{-} } & =\frac{1}{2}(|x|-x) ;
\end{aligned}
$$

$C([0, \omega] ; R)$ is a Banach space of continuous functions $u:[0, \omega] \rightarrow R$ with the norm

$$
\|u\|_{C}=\max \{|u(t)|: t \in[0, \omega]\}
$$

$\widetilde{C}^{2}([0, \omega] ; R)$ is a set of functions $u:[0, \omega] \rightarrow R$ which are absolutely continuous together with their first and second derivatives;

${ }^{*}$ The research was supported by the Academy of Sciences of the Czech Republic, Institutional Research Plan No. AV0Z10190503 and the Grant No. 201/06/0254 of the Grant Agency of the Czech Republic. 
$L([0, \omega] ; R)$ is a Banach space of Lebesgue integrable functions $p:[0, \omega] \rightarrow R$ with the norm

$$
\|p\|_{L}=\int_{0}^{\omega}|p(s)| d s
$$

$\mathcal{P}$ is a set of linear nondecreasing operators $\ell: C([0, \omega] ; R) \rightarrow L([0, \omega] ; R)$, i.e., such linear operators that $\ell(u)(t) \geq 0$ for $t \in[0, \omega]$ whenever $u(t) \geq 0$ for $t \in[0, \omega]$.

The equalities and inequalities between functions are understood almost everywhere in an appropriate interval.

In the present paper, we investigate the question on the existence, uniqueness, and nonnegativity of a solution of the equation ( 0.1$)$ satisfying the boundary conditions

$$
u^{(i)}(0)=u^{(i)}(\omega), \quad i=0,1,2 .
$$

The periodic boundary-value problem for higher order ordinary differential equations has been investigated by many authors (see, e.g., [1-11] and references therein). Note that in [5], unlike the earlier known results, there are investigated, among others, the existence and uniqueness of an $\omega$-periodic solution of the nonautonomous ordinary differential equation

$$
u^{(n)}=\sum_{k=1}^{n} p_{k}(t) u^{(k-1)}+q(t)
$$

without the requirement on the function $p_{1}$ to be of constant sign. In this paper we improve the result of [5] for $n=3$ and $p_{k} \equiv 0, k=2,3$, in a certain way (see Corollary 1.1). For functional differential equations, one can name only a few papers devoted to the study of the periodic boundary-value problem (see, e.g., [12-16]).

The paper is organized as follows. In Section 1, theorems on the existence and uniqueness, as well as on the nonnegativity, of a solution of $(0.1),(0.2)$ are established. Sections 2 and 3 are devoted to the proofs of the main results and examples showing their optimality, respectively.

All the results will be concretized for the differential equation with deviating argument of the form

$$
u^{\prime \prime \prime}(t)=p(t) u(\tau(t))+q(t)
$$

where $p, q \in L([0, \omega] ; R)$ and $\tau:[0, \omega] \rightarrow[0, \omega]$ is a measurable function.

Together with the equation $(0.1)$ we will consider the corresponding homogeneous equation

$$
u^{\prime \prime \prime}(t)=\ell(u)(t)
$$

From the general theory of boundary-value problems for linear functional differential equations, the following theorem is well-known (see [17], for equations with regular operators see, e.g., [18, 19]).

Theorem 0.1. The problem (0.1), (0.2) is uniquely solvable if and only if the corresponding homogeneous problem (0.4), (0.2) has only a trivial solution. 


\section{Main results.}

Theorem 1.1. Let the operator $\ell$ admit the representation $\ell=\ell_{0}-\ell_{1}$, where $\ell_{0}$, $\ell_{1} \in \mathcal{P}$. Let, moreover, $i \in\{0,1\}$, and

$$
\begin{gathered}
\int_{0}^{\omega} \ell_{0}(1)(s) d s+\int_{0}^{\omega} \ell_{1}(1)(s) d s \neq 0, \\
\int_{0}^{\omega} \ell_{i}(1)(s) d s<\frac{32}{\omega^{2}}, \\
\frac{\int_{0}^{\omega} \ell_{i}(1)(s) d s}{1-\frac{\omega^{2}}{32} \int_{0}^{\omega} \ell_{i}(1)(s) d s} \leq \int_{0}^{\omega} \ell_{1-i}(1)(s) d s, \\
\int_{0}^{\omega} \ell_{1-i}(1)(s) d s \leq \frac{64}{\omega^{2}}\left(1+\sqrt{1-\frac{\omega^{2}}{32}} \int_{0}^{\omega} \ell_{i}(1)(s) d s\right.
\end{gathered} .
$$

Then the problem (0.1), (0.2) has a unique solution.

The following two assertions immediately follow from Theorem 1.1.

Corollary 1.1. Let $p \not \equiv 0$, and either

$$
\begin{gathered}
\int_{0}^{\omega}[p(s)]_{+} d s<\frac{32}{\omega^{2}}, \\
\frac{\int_{0}^{\omega}[p(s)]_{+} d s}{1-\frac{\omega^{2}}{32} \int_{0}^{\omega}[p(s)]_{+} d s} \leq \int_{0}^{\omega}[p(s)]_{-} d s \leq \frac{64}{\omega^{2}}\left(1+\sqrt{\left.1-\frac{\omega^{2}}{32} \int_{0}^{\omega}[p(s)]_{+} d s\right)},\right.
\end{gathered}
$$

or

$$
\begin{gathered}
\int_{0}^{\omega}[p(s)]_{-} d s<\frac{32}{\omega^{2}}, \\
\frac{\int_{0}^{\omega}[p(s)]_{-} d s}{1-\frac{\omega^{2}}{32} \int_{0}^{\omega}[p(s)]_{-} d s} \leq \int_{0}^{\omega}[p(s)]_{+} d s \leq \frac{64}{\omega^{2}}\left(1+\sqrt{\left.1-\frac{\omega^{2}}{32} \int_{0}^{\omega}[p(s)]_{-} d s\right)} .\right.
\end{gathered}
$$

Then the problem (0.3), (0.2) has a unique solution.

ISSN 1027-3190. Укр. мат. журн., 2008, m. 60, № 3 
Corollary 1.2. Let $\sigma p(t) \geq 0$ for $t \in[0, \omega]$, where $\sigma \in\{-1,1\}$, and

$$
0<\int_{0}^{\omega}|p(s)| d s \leq \frac{128}{\omega^{2}} .
$$

Then the problem (0.3), (0.2) has a unique solution.

Remark 1.1. The inequality

$$
\int_{0}^{\omega}|p(s)| d s \leq \frac{128}{\omega^{2}}
$$

in Corollary 1.2 is optimal and it cannot be weakened (see Example 3.1 in Section 3).

Theorem 1.2. Let $q(t) \geq 0$ for $t \in[0, \omega]$ and let the operator $\ell$ admit the representation $\ell=\ell_{0}-\ell_{1}$, where $\ell_{0}, \ell_{1} \in \mathcal{P}$. Let, moreover, $i \in\{0,1\}$, the conditions (1.1)-(1.3) be fulfilled, and

$$
\int_{0}^{\omega} \ell_{1-i}(1)(s) d s \leq \frac{32}{\omega^{2}} .
$$

Then the problem $(0.1),(0.2)$ has a unique solution $u$, and

$$
(-1)^{i} u(t) \geq 0 \quad \text { for } \quad t \in[0, \omega] .
$$

Theorem 1.2 implies the following three assertions.

Corollary 1.3. Let $q(t) \geq 0$ for $t \in[0, \omega], p \not \equiv 0$, and

$$
\begin{gathered}
\int_{0}^{\omega}[p(s)]_{+} d s<\frac{32}{\omega^{2}}, \\
\frac{\int_{0}^{\omega}[p(s)]_{+} d s}{1-\frac{\omega^{2}}{32} \int_{0}^{\omega}[p(s)]_{+} d s} \leq \int_{0}^{\omega}[p(s)]_{-} d s \leq \frac{32}{\omega^{2}} .
\end{gathered}
$$

Then the problem (0.3), (0.2) has a unique solution, and this solution is nonnegative.

Corollary 1.4. Let $q(t) \geq 0$ for $t \in[0, \omega], p \not \equiv 0$, and

$$
\begin{gathered}
\int_{0}^{\omega}[p(s)]_{-} d s<\frac{32}{\omega^{2}}, \\
\frac{\int_{0}^{\omega}[p(s)]_{-} d s}{1-\frac{\omega^{2}}{32} \int_{0}^{\omega}[p(s)]_{-} d s} \leq \int_{0}^{\omega}[p(s)]_{+} d s \leq \frac{32}{\omega^{2}} .
\end{gathered}
$$

Then the problem (0.3), (0.2) has a unique solution, and this solution is nonpositive. 
Corollary 1.5. Let $q(t) \geq 0, \sigma p(t) \geq 0$ for $t \in[0, \omega]$, where $\sigma \in\{-1,1\}$, and

$$
0<\int_{0}^{\omega}|p(s)| d s \leq \frac{32}{\omega^{2}}
$$

Then the problem (0.3), (0.2) has a unique solution $u$, and the function $\sigma u$ is nonpositive.

Remark 1.2. The inequality

$$
\int_{0}^{\omega}|p(s)| d s \leq \frac{32}{\omega^{2}}
$$

in Corollary 1.5 is optimal and it cannot be weakened (see Example 3.2 in Section 3).

2. Proofs. To prove Theorems 1.1 and 1.2, we will need the following two lemmas. Lemma 2.1 can be found in [20] in more general form.

Lemma 2.1. Let $u \in \widetilde{C}^{2}([0, \omega] ; R)$ be a nonconstant function satisfying (0.2). Then

$$
M_{0}-m_{0}<\frac{\omega^{2}}{32}\left(M_{2}-m_{2}\right)
$$

where

$$
M_{i}=\max \left\{u^{(i)}(t): t \in[0, \omega]\right\}, \quad m_{i}=\min \left\{u^{(i)}(t): t \in[0, \omega]\right\}, \quad i=0,2 .
$$

Proof. It is obvious that

$$
M_{2}>0, \quad m_{2}<0, \quad M_{0}-m_{0}>0
$$

Put

$$
v(t)= \begin{cases}u(t) & \text { for } t \in[0, \omega] \\ u(t-\omega) & \text { for } t \in] \omega, 2 \omega]\end{cases}
$$

Then, obviously, there exist $a \in[0, \omega[$ and $c \in] a, a+\omega[$ such that

$$
v(a)=m_{0}, \quad v(c)=M_{0} .
$$

The integration by parts on $[a, c]$ yields

$$
M_{0}-m_{0}=\frac{1}{2} \int_{a}^{c}[(c-s)(s-a)]^{\prime} v^{\prime \prime}(s) d s
$$

whence, in view of the continuity of $v^{\prime \prime}$, we get

$$
\begin{gathered}
M_{0}-m_{0}<\frac{1}{2}\left(M_{2} \int_{a}^{\frac{a+c}{2}}[(c-s)(s-a)]^{\prime} d s+m_{2} \int_{\frac{a+c}{2}}^{c}[(c-s)(s-a)]^{\prime} d s\right)= \\
=\frac{(c-a)^{2}}{8}\left(M_{2}-m_{2}\right) .
\end{gathered}
$$

ISSN 1027-3190. Укр. мат. журн., 2008, m. 60, № 3 
Analogously, on the interval $[c, a+\omega]$ we obtain

$$
M_{0}-m_{0}<\frac{(a+\omega-c)^{2}}{8}\left(M_{2}-m_{2}\right) .
$$

Therefore, multiplying the corresponding sides of the inequalities (2.2) and (2.3), on account of the fact that $4 A B \leq(A+B)^{2}$, we have that (2.1) holds.

The lemma is proved.

Lemma 2.2. Let the operator $\ell$ admit the representation $\ell=\ell_{0}-\ell_{1}$, where $\ell_{0}$, $\ell_{1} \in \mathcal{P}$. Let, moreover, $i \in\{0,1\}$ and (1.1)-(1.3) be fulfilled. Then every nontrivial function $u \in \widetilde{C}^{2}([0, \omega] ; R)$ satisfying $(0.2)$ and

$$
(-1)^{i} u^{\prime \prime \prime}(t) \geq(-1)^{i} \ell(u)(t) \quad \text { for } \quad t \in[0, \omega],
$$

assumes positive values.

Proof. Assume on the contrary that there exists a nontrivial nonpositive function $u \in \widetilde{C}^{2}([0, \omega] ; R)$ satisfying $(0.2)$ and (2.4).

First we will show that $u$ is not a constant function. Indeed, supposing $u(t)=$ $=$ const $<0$ for $t \in[0, \omega]$, the integration of (2.4) from 0 to $\omega$ yields

$$
\int_{0}^{\omega} \ell_{1-i}(1)(s) d s \leq \int_{0}^{\omega} \ell_{i}(1)(s) d s,
$$

which contradicts (1.1) and (1.3).

Now put

$$
\begin{gathered}
M_{0}=\max \{|u(t)|: t \in[0, \omega]\}, \quad m_{0}=\min \{|u(t)|: t \in[0, \omega]\}, \\
M_{2}=\max \left\{u^{\prime \prime}(t): t \in[0, \omega]\right\}, \quad m_{2}=-\min \left\{u^{\prime \prime}(t): t \in[0, \omega]\right\},
\end{gathered}
$$

and choose $t_{1}, t_{2} \in[0, \omega]$ such that

$$
u^{\prime \prime}\left(t_{1}\right)=M_{2}, \quad u^{\prime \prime}\left(t_{2}\right)=-m_{2} .
$$

Obviously,

$$
M_{0}>0, \quad m_{0} \geq 0, \quad M_{2}>0, \quad m_{2}>0,
$$

and either

$$
t_{1}<t_{2}
$$

or

$$
t_{1}>t_{2}
$$

Let (2.9) hold. Then the integration of (2.4) from 0 to $t_{1}$, from $t_{1}$ to $t_{2}$, and from $t_{2}$ to $\omega$, respectively, on account of (2.5), (2.7), (2.8), and the assumption $\ell_{0}, \ell_{1} \in \mathcal{P}$, results in

$$
(-1)^{i}\left(M_{2}-u^{\prime \prime}(0)\right) \geq(-1)^{i} \int_{0}^{t_{1}} \ell(u)(s) d s \geq-M_{0} \int_{0}^{t_{1}} \ell_{i}(1)(s) d s,
$$




$$
\begin{aligned}
& (-1)^{i}\left(-m_{2}-M_{2}\right) \geq(-1)^{i} \int_{t_{1}}^{t_{2}} \ell(u)(s) d s \geq-M_{0} \int_{t_{1}}^{t_{2}} \ell_{i}(1)(s) d s, \\
& (-1)^{i}\left(u^{\prime \prime}(\omega)+m_{2}\right) \geq(-1)^{i} \int_{t_{2}}^{\omega} \ell(u)(s) d s \geq-M_{0} \int_{t_{2}}^{\omega} \ell_{i}(1)(s) d s .
\end{aligned}
$$

If $i=0$, then from (2.12) we get

$$
M_{2}+m_{2} \leq M_{0} \int_{0}^{\omega} \ell_{0}(1)(s) d s .
$$

If $i=1$, then from (2.11) and (2.13), in view of (0.2), we obtain

$$
M_{2}+m_{2} \leq M_{0}\left(\int_{0}^{t_{1}} \ell_{1}(1)(s) d s+\int_{t_{2}}^{\omega} \ell_{1}(1)(s) d s\right) \leq M_{0} \int_{0}^{\omega} \ell_{1}(1)(s) d s .
$$

Consequently, we have

$$
M_{2}+m_{2} \leq M_{0} \int_{0}^{\omega} \ell_{i}(1)(s) d s
$$

Analogously one can show that (2.14) is satisfied also in the case when (2.10) holds.

Thus, in both cases (2.9) and (2.10), the inequality (2.14) holds and, according to Lemma 2.1, from (2.14) we get

$$
M_{0}-m_{0}<\frac{\omega^{2}}{32} M_{0} \int_{0}^{\omega} \ell_{i}(1)(s) d s .
$$

On the other hand, the integration of (2.4) from 0 to $\omega$, by virtue of (0.2), (2.5), and the assumption $\ell_{0}, \ell_{1} \in \mathcal{P}$, yields

$$
m_{0} \int_{0}^{\omega} \ell_{1-i}(1)(s) d s \leq M_{0} \int_{0}^{\omega} \ell_{i}(1)(s) d s
$$

From (1.1) and (1.3) it follows that $\int_{0}^{\omega} \ell_{1-i}(1)(s) d s \neq 0$, and thus (2.15) results in

$$
\left(M_{0}-m_{0}\right) \int_{0}^{\omega} \ell_{1-i}(1)(s) d s<\frac{\omega^{2}}{32} M_{0} \int_{0}^{\omega} \ell_{i}(1)(s) d s \int_{0}^{\omega} \ell_{1-i}(1)(s) d s .
$$

Now, using (2.16) in (2.17), on account of (2.8), we have

$$
\int_{0}^{\omega} \ell_{1-i}(1)(s) d s-\int_{0}^{\omega} \ell_{i}(1)(s) d s<\frac{\omega^{2}}{32} \int_{0}^{\omega} \ell_{i}(1)(s) d s \int_{0}^{\omega} \ell_{1-i}(1)(s) d s,
$$

which, in view of (1.2), contradicts (1.3).

The lemma is proved. 
Proof of Theorem 1.1. According to Theorem 0.1 it is sufficient to show that the problem $(0.4),(0.2)$ has only a trivial solution.

Suppose on the contrary that there exists a nontrivial solution $u$ of $(0.4),(0.2)$. Then, according to Lemma 2.2, $u$ assumes both positive and negative values. Put

$$
M_{0}=\max \{u(t): t \in[0, \omega]\}, \quad m_{0}=-\min \{u(t): t \in[0, \omega]\},
$$

and define numbers $M_{2}$ and $m_{2}$ by (2.6). Choose $t_{1}, t_{2} \in[0, \omega]$ such that (2.7) holds. Obviously,

$$
M_{0}>0, \quad m_{0}>0, \quad M_{2}>0, \quad m_{2}>0,
$$

and without loss of generality we can assume that (2.9) hold.

The integration of (0.4) from 0 to $t_{1}$, from $t_{1}$ to $t_{2}$, and from $t_{2}$ to $\omega$, respectively, in view of (2.7), (2.18), (2.19), and the assumption $\ell_{0}, \ell_{1} \in \mathcal{P}$, yields

$$
\begin{gathered}
M_{2}-u^{\prime \prime}(0) \leq M_{0} \int_{0}^{t_{1}} \ell_{0}(1)(s) d s+m_{0} \int_{0}^{t_{1}} \ell_{1}(1)(s) d s, \\
M_{2}+m_{2} \leq m_{0} \int_{t_{1}}^{t_{2}} \ell_{0}(1)(s) d s+M_{0} \int_{t_{1}}^{t_{2}} \ell_{1}(1)(s) d s, \\
u^{\prime \prime}(\omega)+m_{2} \leq M_{0} \int_{t_{2}}^{\omega} \ell_{0}(1)(s) d s+m_{0} \int_{t_{2}}^{\omega} \ell_{1}(1)(s) d s .
\end{gathered}
$$

If we sum the corresponding sides of (2.20) and (2.22), on account of (0.2), we get

$$
M_{2}+m_{2} \leq M_{0} \int_{I} \ell_{0}(1)(s) d s+m_{0} \int_{I} \ell_{1}(1)(s) d s,
$$

where $I=\left[0, t_{1}\right] \cup\left[t_{2}, \omega\right]$. According to Lemma 2.1, from (2.21) and (2.23) we obtain

$$
\begin{aligned}
& M_{0}+m_{0}<\frac{\omega^{2}}{32}\left(m_{0} A_{0}+M_{0} A_{1}\right), \\
& M_{0}+m_{0}<\frac{\omega^{2}}{32}\left(m_{0} B_{1}+M_{0} B_{0}\right),
\end{aligned}
$$

where

$$
\begin{array}{ll}
A_{0}=\int_{t_{1}}^{t_{2}} \ell_{0}(1)(s) d s, & A_{1}=\int_{t_{1}}^{t_{2}} \ell_{1}(1)(s) d s, \\
B_{0}=\int_{I} \ell_{0}(1)(s) d s, & B_{1}=\int_{I} \ell_{1}(1)(s) d s .
\end{array}
$$

If $i=0$, then from (2.24) and (2.25), in view of (1.2) and (2.19), we get 


$$
\begin{aligned}
& 0<m_{0}\left(1-\frac{\omega^{2}}{32} A_{0}\right)<M_{0}\left(\frac{\omega^{2}}{32} A_{1}-1\right) \\
& 0<M_{0}\left(1-\frac{\omega^{2}}{32} B_{0}\right)<m_{0}\left(\frac{\omega^{2}}{32} B_{1}-1\right),
\end{aligned}
$$

whence we obtain

$$
\left(1-\frac{\omega^{2}}{32} A_{0}\right)\left(1-\frac{\omega^{2}}{32} B_{0}\right)<\left(\frac{\omega^{2}}{32} A_{1}-1\right)\left(\frac{\omega^{2}}{32} B_{1}-1\right)
$$

If $i=1$, then from (2.24) and (2.25), in view of (1.2) and (2.19), we get

$$
\begin{aligned}
& 0<M_{0}\left(1-\frac{\omega^{2}}{32} A_{1}\right)<m_{0}\left(\frac{\omega^{2}}{32} A_{0}-1\right), \\
& 0<m_{0}\left(1-\frac{\omega^{2}}{32} B_{1}\right)<M_{0}\left(\frac{\omega^{2}}{32} B_{0}-1\right),
\end{aligned}
$$

whence we obtain

$$
\left(1-\frac{\omega^{2}}{32} A_{1}\right)\left(1-\frac{\omega^{2}}{32} B_{1}\right)<\left(\frac{\omega^{2}}{32} A_{0}-1\right)\left(\frac{\omega^{2}}{32} B_{0}-1\right) .
$$

Consequently, we have

$$
\left(1-\frac{\omega^{2}}{32} A_{i}\right)\left(1-\frac{\omega^{2}}{32} B_{i}\right)<\left(\frac{\omega^{2}}{32} A_{1-i}-1\right)\left(\frac{\omega^{2}}{32} B_{1-i}-1\right) .
$$

On the other hand, on account of (2.26) and (2.27), using the inequality $4 A B \leq$ $\leq(A+B)^{2}$, we find

$$
\begin{gathered}
\left(1-\frac{\omega^{2}}{32} A_{i}\right)\left(1-\frac{\omega^{2}}{32} B_{i}\right) \geq 1-\frac{\omega^{2}}{32} \int_{0}^{\omega} \ell_{i}(1)(s) d s \\
\left(\frac{\omega^{2}}{32} A_{1-i}-1\right)\left(\frac{\omega^{2}}{32} B_{1-i}-1\right) \leq \frac{1}{4}\left(\frac{\omega^{2}}{32} \int_{0}^{\omega} \ell_{1-i}(1)(s) d s-2\right)^{2} .
\end{gathered}
$$

Now, using (2.33) and (2.34) in (2.32), we get

$$
1-\frac{\omega^{2}}{32} \int_{0}^{\omega} \ell_{i}(1)(s) d s<\frac{1}{4}\left(\frac{\omega^{2}}{32} \int_{0}^{\omega} \ell_{1-i}(1)(s) d s-2\right)^{2} .
$$

Moreover, from (2.28)-(2.31), by virtue of (2.19), (2.26), and (2.27), we have

$$
\frac{\omega^{2}}{32} \int_{0}^{\omega} \ell_{1-i}(1)(s) d s \geq \frac{\omega^{2}}{32}\left(A_{1-i}+B_{1-i}\right)>2 .
$$

Therefore, the inequality (2.35), on account of (1.2) and (2.36), contradicts (1.4).

The theorem is proved. 
Proof of Theorem 1.2. According to Theorem 1.1, the problem (0.1), (0.2) has a unique solution $u$. It is sufficient to show that (1.6) holds.

Suppose the contrary, that there exists $t_{0} \in[0, \omega]$ such that $(-1)^{i} u\left(t_{0}\right)<0$. Then, according to Lemma 2.2, the function $u$ assumes both positive and negative values. Define numbers $M_{0}, m_{0}, M_{2}$, and $m_{2}$ by (2.18) and (2.6), respectively, and choose $t_{1}, t_{2} \in[0, \omega]$ such that (2.7) is fulfilled. Obviously, (2.19) holds.

Let (2.9) be satisfied. Then the integration of (0.1) from $t_{1}$ to $t_{2}$, in view of (2.7), (2.18), and the assumptions $\ell_{0}, \ell_{1} \in \mathcal{P}$ and $q(t) \geq 0$ for $t \in[0, \omega]$, results in

$$
\begin{aligned}
& m_{2}+M_{2}=-\int_{t_{1}}^{t_{2}}(\ell(u)(s)+q(s)) d s \leq \\
& \leq m_{0} \int_{0}^{\omega} \ell_{0}(1)(s) d s+M_{0} \int_{0}^{\omega} \ell_{1}(1)(s) d s .
\end{aligned}
$$

Let (2.10) be satisfied. Then the integration of (0.1) from 0 to $t_{2}$ and from $t_{1}$ to $\omega$, respectively, in view of (2.7), (2.18), and the assumptions $\ell_{0}, \ell_{1} \in \mathcal{P}$ and $q(t) \geq 0$ for $t \in[0, \omega]$, yields

$$
\begin{aligned}
& m_{2}+u^{\prime \prime}(0)=-\int_{0}^{t_{2}}(\ell(u)(s)+q(s)) d s \leq \\
& \leq m_{0} \int_{0}^{t_{2}} \ell_{0}(1)(s) d s+M_{0} \int_{0}^{t_{2}} \ell_{1}(1)(s) d s, \\
& M_{2}-u^{\prime \prime}(\omega)=-\int_{t_{1}}^{\omega}(\ell(u)(s)+q(s)) d s \leq \\
& \leq m_{0} \int_{t_{1}}^{\omega} \ell_{0}(1)(s) d s+M_{0} \int_{t_{1}}^{\omega} \ell_{1}(1)(s) d s .
\end{aligned}
$$

If we sum the corresponding sides of the last two inequalities, on account of (0.2) we obtain

$$
m_{2}+M_{2} \leq m_{0} \int_{0}^{\omega} \ell_{0}(1)(s) d s+M_{0} \int_{0}^{\omega} \ell_{1}(1)(s) d s .
$$

Thus, in both cases (2.9) and (2.10) we have (2.37).

Now, according to Lemma 2.1, the inequality (2.37) results in

$$
M_{0}+m_{0}<\frac{\omega^{2}}{32}\left(m_{0} \int_{0}^{\omega} \ell_{0}(1)(s) d s+M_{0} \int_{0}^{\omega} \ell_{1}(1)(s) d s\right)
$$

whence, on account of (1.2) and (1.5), we get a contradiction $M_{0}+m_{0}<M_{0}+m_{0}$.

The theorem is proved. 


\section{Examples.}

Example 3.1. Let $\sigma \in\{-1,1\}, \varepsilon>0$ be arbitrary, and choose $k \in] 0,1]$ and $d \in] 0,1 / 4\left[\right.$ such that $384 /\left(\left(3-16 d^{2}\right) k\right)=128+\varepsilon$. Put

$$
x(t)= \begin{cases}\frac{96}{\left(3-16 d^{2}\right) k d} t & \text { for } t \in[0, d[, \\ \frac{96}{\left(3-16 d^{2}\right) k} & \text { for } t \in[d, 1 / 2-d[, \\ \frac{96}{\left(3-16 d^{2}\right) k d}(1 / 2-t) & \text { for } t \in[1 / 2-d, 1 / 2+d[, \\ -\frac{96}{\left(3-16 d^{2}\right) k} & \text { for } t \in[1 / 2+d, 1-d[, \\ \frac{96}{\left(3-16 d^{2}\right) k d}(t-1) & \text { for } t \in[1-d, 1]\end{cases}
$$

and

$$
u(t)=-(1-t) \int_{0}^{t} s x(s) d s-t \int_{t}^{1}(1-s) x(s) d s .
$$

Then the function $u$ satisfies the conditions (0.2) with $\omega=1$, and $u(3 / 4)=-u(1 / 4)=$ $=1 / k$. Therefore we can choose $t_{0}, t_{1} \in[0,1]$ such that $u\left(t_{0}\right)=\sigma, u\left(t_{1}\right)=-\sigma$. Obviously, $u$ is a nontrivial solution of the problem

$$
u^{\prime \prime \prime}(t)=p(t) u(\tau(t)), \quad u^{(i)}(0)=u^{(i)}(1), \quad i=0,1,2,
$$

with

$$
\begin{gathered}
p(t) \stackrel{\text { df }}{=} \sigma\left|x^{\prime}(t)\right| \quad \text { for } \quad t \in[0, \omega], \\
\tau(t) \stackrel{\text { df }}{=}\left\{\begin{array}{lc}
t_{0} & \text { for } t \in[0,1 / 4[\cup[3 / 4,1], \\
t_{1} & \text { for } t \in[1 / 4,3 / 4[.
\end{array}\right.
\end{gathered}
$$

On the other hand, $\sigma p(t) \geq 0$ for $t \in[0, \omega]$ and

$$
\int_{0}^{1}|p(s)| d s=4 \cdot \frac{96}{\left(3-16 d^{2}\right) k}=128+\varepsilon .
$$

Example 3.2. Let $\sigma \in\{-1,1\}, \varepsilon>0$ be arbitrary, and choose $k \in] 0,1[$ and $d \in] 0,1 / 4\left[\right.$ such that $96 /\left(\left(3-16 d^{2}\right) k\right)=32+\varepsilon$. Put 


$$
x(t)= \begin{cases}\frac{48}{\left(3-16 d^{2}\right) k d} t & \text { for } t \in[0, d[, \\ \frac{48}{\left(3-16 d^{2}\right) k} & \text { for } t \in[d, 1 / 2-d[, \\ \frac{48}{\left(3-16 d^{2}\right) k d}(1 / 2-t) & \text { for } t \in[1 / 2-d, 1 / 2+d[, \\ -\frac{48}{\left(3-16 d^{2}\right) k} & \text { for } t \in[1 / 2+d, 1-d[, \\ \frac{48}{\left(3-16 d^{2}\right) k d}(t-1) & \text { for } t \in[1-d, 1]\end{cases}
$$

and

$$
u(t)=-\frac{\sigma}{2}-(1-t) \int_{0}^{t} s x(s) d s-t \int_{t}^{1}(1-s) x(s) d s .
$$

Then the function $u$ satisfies the conditions (0.2) with $\omega=1$, and $u(1 / 4)=-\sigma / 2-$ $-1 /(2 k)<-(\sigma+1) / 2, u(3 / 4)=-\sigma / 2+1 /(2 k)>(1-\sigma) / 2$. Therefore we can choose $t_{0} \in[0,1]$ such that $u\left(t_{0}\right)=-\sigma$. Obviously, $u$ assumes both positive and negative values and $u$ is a solution of the problem

$$
u^{\prime \prime \prime}(t)=p(t) u\left(t_{0}\right)+q(t), \quad u^{(i)}(0)=u^{(i)}(1), \quad i=0,1,2,
$$

with

$$
p(t) \stackrel{\text { df }}{=} \sigma\left[x^{\prime}(t)\right]_{-}, \quad q(t) \stackrel{\text { df }}{=}\left[x^{\prime}(t)\right]_{+} \quad \text { for } \quad t \in[0, \omega] .
$$

On the other hand, $q(t) \geq 0, \sigma p(t) \geq 0$ for $t \in[0, \omega]$, and

$$
\int_{0}^{1}|p(s)| d s=2 \cdot \frac{48}{\left(3-16 d^{2}\right) k}=32+\varepsilon .
$$

1. Baslandze S. R., Kiguradze I. T. On the unique solvability of a periodic boundary value problem for third-order linear differential equations // Differents. Uravneniya. - 2006. - 42, № 2. - P. 153-158.

2. Cabada A. The method of lower and upper solutions for second, third, fourth, and higher order boundary value problems // J. Math. Anal. and Appl. - 1994. - 185. - P. 302-320.

3. Cabada A. The method of lower and upper solutions for third order periodic boundary value problems // Ibid. - 1995. - 195. - P. $568-589$.

4. Gegelia G. T. On boundary value problems of periodic type for ordinary odd order differential equations // Arch. Math. - 1984. - 4. - P. $195-204$.

5. Kiguradze I. On periodic solutions of $n$-th order ordinary differential equations // Nonlinear Anal.: Theory, Meth. and Appl. - 2000. - 40. - P. 309-321.

6. Kiguradze I. T., Kusano T. On periodic solutions of higher order nonautonomous ordinary differential equations // Differents. Uravneniya. - 1999. - 35, № 1. - P. $72-78$.

7. Lasota A., Opial Z. Sur les solutions périodiques des équations différentielles ordinaires // Ann. polon. math. - 1964. - 16. - P. $69-94$.

8. Lasota A., Szafraniec F. $H$. Sur les solutions périodiques d'une équation différentielle ordinaire d'ordre $n$ // Ibid. - 1966. - 18. - P. $339-344$.

9. Nieto J. J. Periodic solutions for third order ordinary differential equations // Comment. math. Univ. carol. - 1991. - 32, № 3. - P. 495 - 499. 
10. Omari P., Trombetta $M$. Remarks on the lower and upper solutions method for second- and third-order periodic boundary value problems // Appl. Math. Comput. - 1992. - 50, № 1. - P. 1 - 21.

11. Villari $G$. Soluzioni periodiche di una classe di equazioni differenziali del terz'ordine quasi lineari // Ann. mat. pura ed appl. - 1966. - 73, № 4. - P. 103 - 110.

12. Lomtatidze A., Mukhigulashvili S. On periodic solutions of second order functional differential equations // Mem. Different. Equat. Math. Phys. - 2004. - 5. - P. 125 - 126.

13. Mukhigulashvili $S$. On a periodic boundary value problem for second-order linear functional differential equations // Boundary Value Problems. - 2005. - № 3. - P. 247-261.

14. Mukhigulashvili S., Půža B. On a periodic boundary value problem for third order linear functional differential equations // Funct. Different. Equat. (to appear).

15. Ronto A. N. On the periods of periodic motions in autonomous systems // Ukr. Math. J. - 2001. 53, № 1. - P. $108-128$.

16. Ronto A. N., Samoilenko A. M. On the unique solvability of linear boundary value problems of periodic type // Dopov. Nats. Akad. Nauk Ukr. - 2002. - № 9. - P. 42 - 46.

17. Hakl R., Mukhigulashvili $S$. On a boundary value problem for $n$-th order linear functional differential systems // Georg. Math. J. - 2005. - 12, № 2. - P. $229-236$.

18. Kiguradze I., Püža B. On boundary value problems for systems of linear functional differential equations // Czech. Math. J. - 1997. - 47, № 2. - P. $341-373$.

19. Schwabik $\check{S}$., Tvrdý M., Vejvoda $O$. Differential and integral equations: boundary value problems and adjoints. - Praha: Academia, 1979.

20. Hakl R., Mukhigulashvili S. On one estimate for periodic functions // Georg. Math. J. - 2005. - 12, № 1. - P. $97-114$.

Received 15.10.07 